\title{
Características morfológicas de las cianobacterias y fitoplancton dominante en embalses de Antioquia: un enfoque basado en el biovolumen
}

\section{Morphological characteristics of cyanobacteria and dominant phytoplankton in reservoirs in Antioquia: a focus based in biovolume}

Karen Palacio Gómez ${ }^{1 *}$; Esnedy Hernández Atilano²; Gustavo Peñuela Mesa ${ }^{3}$; Néstor Aguirre Ramírez ${ }^{4}$; Fabio Vélez Macías ${ }^{5}$

1'Ing. Ambiental. Universidad de Antioquia. Medellín, Antioquia, Colombia; e-mail: karen.palacio@udea.edu.co; Dhttps://orcid.org/0000-0003-2671-9294

²Bióloga, Doctora. Universidad de Antioquia. Medellín, Antioquia, Colombia; e-mail: esnedy.hernandez@udea.edu.co; (Dhttps://orcid.org/0000-0003-23801436

${ }^{3}$ Químico, Ph.D. Universidad de Antioquia, Grupo de Diagnóstico y Control de la Contaminación (GDCON). Medellín, Antioquia, Colombia; e-mail: gustavo. penuela@udea.edu.co; Dhttps://orcid.org/0000-0003-3065-0285

${ }^{4}$ Licenciado en Biología y Química, Ph.D. Universidad de Antioquia. Medellín, Antioquia, Colombia; e-mail: nestor.aguirre@udea.edu.co; Dhttp://orcid. org/0000-0001-7901-0479

${ }^{5}$ Ing. Sanitario, Ph.D. Universidad de Antioquia, Grupo de Investigación GeoLimna. Medellín, Antioquia, Colombia; e-mail: fabio.velez@udea.edu.co; (Dhttps://orcid.org/0000-0001-6348-6405

*autor de correspondencia: karen.palacio@udea.edu.co

Cómo citar: Palacio Gómez, K.; Hernández Atilano, E.; Peñuela Mesa, G.; Aguirre Ramírez, N.; Vélez Macías, F. 2019. Características morfológicas de las cianobacterias y fitoplancton dominante en embalses de Antioquia: un enfoque basado en el biovolumen. Rev. U.D.C.A Act. \& Div. Cient. 22(2):e1306. http://doi.org/10.31910/rudca.v22.n2.2019.1306

Artículo de acceso abierto publicado por Revista U.D.C.A Actualidad \& Divulgación Científica, bajo una licencia Creative Commons CC BY-NC 4.0

Recibido: Julio 27 de 2018

Aceptado: Julio 16 de 2019

Editado por: Ingeborg Zenner de Polanía

\section{RESUMEN}

El desarrollo masivo de cianobacterias y la contaminación por cianotoxinas constituyen una problemática ambiental de especial interés en los sistemas acuáticos, debido a los diversos impactos negativos que los florecimientos (blooms) de cianobacterias pueden ocasionar. Dada la importancia recreacional y de abastecimiento de los embalses Abreo - Malpaso, Peñol - Guatapé y Playas, ubicados en el oriente de Antioquia - Colombia, en este estudio, se estimó la densidad de cianobacterias planctónicas y otros organismos del fitoplancton, así como el biovolumen geométrico, la relación superficie volumen $(\mathrm{S} / \mathrm{V})$ y la dimensión lineal máxima (DLM), a través de cuatro muestreos, en tres estaciones, para cada uno de los embalses. Se identificaron, dentro del phylum Cyanobacteria, con potencial de producción de cianotoxinas, el complejo Microcystis, los géneros Woronichinia, Aphanocapsa y Oscillatoria y la especie Radiocystis fernandoi. Al aplicar la prueba no parámetrica Kruskal - Wallis, no se encontraron diferencias significativas entre los embalses estudiados ( $>>0,05)$; sin embargo, el embalse El Peñol - Guatapé presentó la mayor biomasa algal y densidad de cianobacterias, por lo que, según 
los niveles de riesgo planteados para los embalses estudiados y de acuerdo con las características morfológicas de estos organismos, este embalse presenta un nivel de riesgo Medio Alto con relación a la presencia y morfología de las cianobacterias, mientras que los embalses Abreo - Malpaso y Playas están asociados a un riesgo Medio Bajo. Se sugiere establecer medidas de control y de prevención, con el fin de evitar un desarrollo masivo de cianobacterias, una potencial presencia de cianotoxinas y afectaciones graves a seres humanos y animales.

Palabras clave: morfología; riesgo; fitoplancton; biovolumen; embalse.

\section{ABSTRACT}

Cyanotoxin contamination is of special interest in water bodies, especially those for human use, due to the various negative effects that blooms of potentially toxic cyanobacteria can cause on human health. Given the recreational and supply importance of the Abreo - Malpaso, Peñol - Guatapé and Playas reservoirs in the Department of Antioquia, Colombia. This study has estimated the biomass of the planktonic cyanobacteria, their geometric biovolume, the surface volume ratio $(\mathrm{S} / \mathrm{V}$ ) and the maximum linear dimension (MLD), during four sampling seasons in three different stations for each of these bodies. Water. We have identified from the Cyanophyta division with the production of cyanotoxins, the Microcystis complex and generates Woronichinia, Aphanocapsa and Oscillatoria, as well as the species Radiocystis fernandoi. When applying the nonparametric Kruskal - Wallis test, no significant differences were found between the studied reservoirs ( $p>0.05)$; however, the Peñol - Guatapé reservoir presented the highest algae biomass and density of cyanobacteria, therefore, according to the levels of risk posed for the reservoirs studied and according to the morphological characteristics of these organisms, this reservoir presents a High Medium risk level in relation to the presence and morphology of the cyanobacteria, while the Abreo-Malpaso and Playas reservoirs are associated with a Medium Low risk. It is suggested to establish control and prevention measures to avoid a massive development of cyanobacteria, a possible presence of cyanotoxins and serious affectations in humans and animals.

Keywords: morphology; risk; phytoplankton; biovolume; reservoir.

\section{INTRODUCCIÓN}

Las cianobacterias o algas verde-azules son los organismos fotosintetizadores aeróbicos más antiguos del planeta; algunos pueden fijar nitrógeno molecular, mientras que otros pueden incorporar y almacenar fósforo, contribuyendo significativamente a la producción primaria acuática (Bonilla et al. 2015). Estos organismos presentan una gran plasticidad morfológica y adaptabilidad a diversos tipos de ecosistemas, características que les confieren una ventaja para colonizar exitosamente diferentes sistemas acuáticos, por lo que se encuentran ampliamente distribuidos en el mundo (Otten \& Paerl, 2015).
Esta importante capacidad para colonizar diversos sistemas favorece su desarrollo masivo, propiciando la formación de "blooms", los cuales, pueden conferirle aspectos alterados al agua, como cambios en la coloración o formación de espuma (Rolland et al. 2013) y producción de olores y sabores desagradables (Perovich et al. 2008), generando, finalmente, limitaciones significativas para la potabilización del agua o para fines de recreación (Otten \& Paerl, 2015); sin embargo, el tema de mayor preocupación, a nivel mundial, es su potencial de producción de toxinas, denominadas cianotoxinas, que se pueden acumular en la red trófica, produciendo síntomas de intoxicación en seres humanos y animales (Oberholster et al. 2006).

En Colombia existen reportes de intoxicación por cianotoxinas en aves, en la Ciénaga Grande de Santa Marta (Bula, 1985; Mancera et al. 1994) y la Ciénaga San Rafael de Buenavista (Escobar \& Manjarres, 1985). Igualmente, en diversos humedales del altiplano cundiboyacense, se encontró una importante dominancia de estos organismos (Vásquez et al. 2006).

Dada la importancia recreacional y de abastecimiento de los embalses Abreo - Malpaso, Peñol - Guatapé y Playas, ubicados en el oriente de Antioquia, Colombia, se estimaron diferentes variables físicoquímicas, así como la densidad de cianobacterias planctónicas y otros organismos del fitoplancton, además de variables morfológicas, como biovolumen geométrico, relación superficie volumen $(\mathrm{S} / \mathrm{V})$ y dimensión lineal máxima (DLM), para identificar características morfológicas, especialmente, de las cianobacterias y determinar los niveles de riesgo, con relación al desarrollo masivo de estos organismos.

\section{MATERIALES Y MÉTODOS}

Área de estudio. El área de estudio comprendió tres embalses: Abreo - Malpaso, El Peñol - Guatapé y Playas, ubicados en el oriente del departamento de Antioquia, Colombia. El embalse Abreo - Malpaso, se encuentra en el municipio de Rionegro, en el suroriente del departamento de Antioquia, aproximadamente, a $2.080 \mathrm{~m}$ s.n.m. y un área de tan solo 5,3ha, considerablemente menor, en comparación con los embalses adicionales; su importancia para la región radica en el abastecimiento de gran parte del municipio de Rionegro (CORNARE, 2012). El embalse El Peñol - Guatapé, se localiza en el municipio de Guatapé, a 50km de Medellín y una altitud de $2.000 \mathrm{~m}$ s.n.m., además abarca un área de $6240 \mathrm{ha}$, constituyéndose como uno de los embalses de regulación más grande del país, haciendo parte de una de las cadenas hidráulicas, que más energía eléctrica le aporta al sistema (Aguirre et al. 2007). Finalmente, el embalse Playas, localizado a $2.775 \mathrm{~m}$ s.n.m., bajo la jurisdicción de los municipios de San Rafael y San Carlos, Antioquia, presenta un área aproximada de $790 \mathrm{ha}$, donde se realizan captaciones del agua del río Guatapé y sus afluentes, para alimentar la hidroeléctrica Playas, localizada en el municipio de San Carlos (CORNARE, 2012a).

\section{Diseño de muestreo}

Fitoplancton: Durante el 2015 (noviembre) y el 2016 (febrero, abril y junio) fueron realizadas 4 campañas de muestreo en cada uno de los embalses estudiados, incluyendo 3 estaciones de muestreo en cada 
cuerpo de agua, correspondientes al ingreso del agua al embalse, centro del embalse y la salida de aguas. En cada estación, se tomó una muestra de agua para análisis semicuantitativo de la comunidad fitoplanctónica, mediante arrastres superficiales horizontales, con una red de plancton de $25 \mu \mathrm{m}$ de diámetro de poro, durante 5 minutos, a una velocidad de $0,1 \mathrm{~m} / \mathrm{s}$. El material colectado, se fijó in situ, con solución de Lugol, al 10\%.

Variables físicas y químicas: Para cada una de las estaciones distribuidas en los embalses, se realizaron análisis de parámetros fisicoquímicos in situ y en laboratorio para cada una de las cuatro campañas. In situ fueron medidos transparencia, a través de un disco Secchi; el oxígeno disuelto, el $\mathrm{pH}$ y la conductividad eléctrica fueron calculados, a través de un equipo multiparamétrico Hach (modelo HQ40d) y la turbidez, por medio de turbidímetro Hach (2100Q). En el laboratorio fueron medidas las variables alcalinidad y dureza, por método titulométrico; nitratos $\left(\mathrm{NO}_{3}\right)$, a través de colorimetría; nitrógeno total Kjeldahl (NTK), hierro (Fe), fósforo soluble (PSoluble) y fósforo total (PTotal), por medio de espectofotometría; carbono orgánico disuelto (COD) y carbono orgánico total (COT), a través de oxidación.

Análisis de fitoplancton en laboratorio: Para el análisis de las muestras de fitoplancton, se utilizó un microscopio invertido Boeco (modelo BIB-100). Las observaciones al microscopio, se efectuaron según Ros (1979), por medio de un recuento en 30 campos de observación seleccionados aleatoriamente (Uhelinger, 1964), controlando la estabilidad de conteo, a través de la curva de rarefacción. El conteo, se efectuó con una magnificación total de 400X. Para la identificación taxonómica, se emplearon los trabajos de Hoffmann et al. (2005), Komárek \& Anagnostidis (1989) y Komárek \& Anagnostidis (1999).

Para estimar el biovolumen de las algas planctónicas, se utilizó el método del volumen celular medio, obtenido a partir de las dimensiones de, al menos, 30 células y 30 colonias seleccionadas aleatoriamente en el microscopio, asemejando cada una de estas a sólidos geométricos, empleando la metodología descrita en Hillebrand et al. (1999). La densidad absoluta (cél/mL) de los organismos, así como el promedio del número de células de algas que formaban colonias fue multiplicado por su volumen celular medio, para obtener un estimativo de la biomasa de los organismos (Hernández et al. 2011).

Procesamiento y análisis de la información: Los resultados morfológicos y de biovolumen para el fitoplancton estudiado, se describieron mediante la media aritmética, los valores máximos y mínimos y el coeficiente de variación. Para determinar diferencias significativas de las variables entre los sistemas acuáticos, se aplicó la prueba no paramétrica de Kruskal- Wallis, luego de probar que los datos no provienen de una distribución normal, empleando la prueba Shapiro Wilk $(\mathrm{p}<0,05)$, con el programa STATISTICA 6.0.

Para establecer el grado de relación entre las variables morfológicas (Biovolumen, DLM y relación S/V) y el conjunto de variables ambientales, se ejecutó un análisis de correspondencia sin tendencia
(DCCA) y debido a la longitud del primer gradiente $(\mathrm{AX} 1=0,978)$, se realizó un análisis de redundancia canónica $(\mathrm{RDA})$. Las variables incluidas en dicha prueba fueron Alcalinidad total, Carbono orgánico disuelto, Carbono orgánico total, dureza total, dureza cálcica, Hierro disuelto, Nitrógeno amoniacal, Nitrógeno total Kjeldahl, Nitratos, Fósforo soluble, Fósforo total, transparencia, temperatura, $\mathrm{pH}$, Oxígeno disuelto, conductividad y turbiedad, de las cuales, todas registraron un factor de inflación menor a 20; esta prueba, se realizó empleando el programa R. Previo a este abordaje, las variables fueron estandarizadas (xmin/max-min) y transformadas a su raíz cuadrada $(\mathrm{vx}+1)$.

\section{RESULTADOS Y DISCUSIÓN}

A pesar de la conexión existente entre los tres sistemas acuáticos estudiados, los embalses Abreo - Malpaso, El Peñol - Guatapé y Playas, exhiben una variación en relación a la abundancia, la taxonomía y la morfología de los organismos fitoplanctónicos encontrados, especialmente, el phylum Cyanobacteria, así como una importante diferencia en los parámetros fisicoquímicos y características limnológicas; dichas características, en los tres cuerpos de agua estudiados, estuvieron fuertemente relacionadas con la morfología del fitoplancton identificado en estos sistemas, sin incluir análisis temporales en el estudio de los datos.

Variables físicas y químicas. Las diferencias en los resultados de las variables analizadas están asociadas, principalmente, a la localización de los sistemas acuáticos. Los resultados obtenidos para las variables fisicoquímicas, se presentan en la tabla 1, de igual forma, los resultados obtenidos con relación a los nutrientes, se indican en la tabla 2.

Los resultados del RDA (Figura 1), para explicar la distribución de las variables morfológicas con relación a las variables ambientales ya mencionadas, generaron un porcentaje de explicación de la varianza total del $64 \%(\mathrm{p}=0,026)$. El esquema indicó que el primer eje positivo $(0,497)$ mostró una relación directa, principalmente, entre los nutrientes evaluados (Ptotal, PSoluble, NTK y NO 3 ) y las variables biológicas estudiadas (Biovolumen, densidad, relación S/V y DLM), mientras que, en el primer eje negativo, se observó una fuerte relación entre las variables COT, Fe total y Temperatura.

La primera estación del embalse El Peñol - Guatapé mostró una importante relación con las diferentes formas de nutrientes analizados, lo que está ligado a la presencia de "blooms" en esta estación, además de estar también relacionado con las variables mayores valores de densidad de organismos, biovolumen y DLM. Algunas de las estaciones correspondientes al embalse Abreo Malpaso presentaron relaciones importantes con las variables COT y Fe disuelto, lo que se puede asociar a los vertimientos realizados en el embalse y el aporte de nutrientes por escorrentía. Por otro lado, un grupo de variables, como alcalinidad y transparencia, se asocian, principalmente, a estaciones del embalse Playas, el sistema acuático con menos perturbaciones de origen antrópico, de los tres embalses estudiados. 
Tabla 1. Valores medios $(\mu)$, máximos y mínimos (max-min) y coeficiente de variación (C.V.) de las variables físicoquímicas, en los tres embalses (AM: Embalse Abreo - Malpaso; PG: Embalse Peñol - Guatapé; PL: Embalse Playas). Alcal: Alcalinidad total (mg $\mathrm{CaCO}_{3} / \mathrm{L}$ ); Transp: Transparencia (cm); Temp: Temperatura ( $\left.{ }^{\circ} \mathrm{C}\right) ; \mathrm{pH}$; OD: Oxígeno disuelto (mg/L); Cond: Conductividad ( $\left.\mu \mathrm{s} / \mathrm{cm}\right)$; Turb: Turbiedad (UNT); DurTot: Dureza total (mg $\left.\mathrm{CaCO}_{3} / \mathrm{L}\right)$; DurCalc: Dureza Calcica (mg $\left.\mathrm{CaCO}_{3} / \mathrm{L}\right)$; COD: Carbono orgánico disuelto (mg C/L); COT: Carbono orgánico total (mg C/L).

\begin{tabular}{|c|c|c|c|c|c|c|c|c|c|c|c|c|}
\hline & & Alca & Transp & Temp & $\mathrm{pH}$ & OD & Cond & Turb & DurTot & DurCalc & COD & COT \\
\hline \multirow{3}{*}{$\mathrm{AM}$} & $\mu$ & 24,86 & 51,83 & 22,26 & 6,30 & 4,93 & 56,73 & 28,66 & 27,80 & 10,67 & 4,30 & 5,18 \\
\hline & Min-max & $\begin{array}{l}(17,93- \\
35,98)\end{array}$ & $\begin{array}{l}(45,00 \\
68,00)\end{array}$ & $\begin{array}{l}(20,30- \\
24,20)\end{array}$ & $\begin{array}{c}(6,19- \\
6,50)\end{array}$ & $\begin{array}{l}(3,37- \\
7,23)\end{array}$ & $\begin{array}{c}(49,10- \\
62,60)\end{array}$ & $\begin{array}{l}(10,21- \\
39,95)\end{array}$ & $\begin{array}{l}(21,90- \\
39,14)\end{array}$ & $\begin{array}{c}(5,1- \\
26,37)\end{array}$ & $\begin{array}{l}(2,00- \\
7,00)\end{array}$ & $\begin{array}{l}(1,40- \\
8,79)\end{array}$ \\
\hline & C.V. & 0,24 & 0,14 & 0,06 & 0,01 & 0,28 & 0,08 & 0,31 & 0,23 & 0,57 & 0,39 & 0,52 \\
\hline \multirow{3}{*}{ PG } & $\mu$ & 16,72 & 233,09 & 17,76 & 7,76 & 7,43 & 51,58 & 27,95 & 16,47 & 8,28 & 3,04 & 3,39 \\
\hline & Min-max & $\begin{array}{c}(13,350- \\
24,80)\end{array}$ & $\begin{array}{l}(23,300- \\
503,00)\end{array}$ & $\begin{array}{l}(7,170- \\
23,900)\end{array}$ & $\begin{array}{l}(6,98- \\
9,35)\end{array}$ & $\begin{array}{l}(6,54- \\
9,44)\end{array}$ & $\begin{array}{l}(39,00- \\
93,70)\end{array}$ & $\begin{array}{l}(0,74- \\
87,77)\end{array}$ & $\begin{array}{l}(7,51- \\
30,93)\end{array}$ & $\begin{array}{l}(2,29- \\
14,27)\end{array}$ & $\begin{array}{l}(1,64- \\
5,05)\end{array}$ & $\begin{array}{l}(1,82- \\
5,92)\end{array}$ \\
\hline & C.V. & 0,216 & 0,879 & 0,360 & 0,099 & 0,140 & 0,313 & 1,281 & 0,391 & 0,374 & 0,353 & 0,357 \\
\hline \multirow{3}{*}{ PL } & $\mu$ & 37,09 & 211,55 & 25,58 & 7,19 & 7,44 & 44,06 & 12,16 & 15,39 & 8,28 & 2,86 & 3,27 \\
\hline & Min-max & $\begin{array}{l}(13,35- \\
106,50)\end{array}$ & $\begin{array}{l}(28,00- \\
444,00)\end{array}$ & $\begin{array}{l}(21,10- \\
29,20)\end{array}$ & $\begin{array}{l}(6,81- \\
7,53)\end{array}$ & $\begin{array}{l}(7,13- \\
7,91)\end{array}$ & $\begin{array}{c}(22,20- \\
67,10)\end{array}$ & $\begin{array}{l}(0,86- \\
63,58)\end{array}$ & $\begin{array}{l}(7,51- \\
27,29)\end{array}$ & $\begin{array}{l}(4,44- \\
14,50)\end{array}$ & $\begin{array}{l}(1,64- \\
3,84)\end{array}$ & $\begin{array}{l}(1,82- \\
5,13)\end{array}$ \\
\hline & C.V. & 0,98 & 0,68 & 0,09 & 0,02 & 0,03 & 0,24 & 1,54 & 0,34 & 0,36 & 0,24 & 0,25 \\
\hline
\end{tabular}

Tabla 2. Valores medios ( $\mu$ ), máximos y mínimos (max-min) y coeficiente de variación (C.V.) de los nutrientes analizados, en los tres embalses (AM: Embalse Abreo - Malpaso; PG: Embalse Peñol - Guatapé; PL: Embalse Playas). FeDisu: Hierro disuelto (mg Fe/L); Namon: Nitrógeno amoniacal (mg NH3-N/L); NTK: Nitrógeno total Kjeldahl (mg N/L); $\mathrm{NO}_{3}$ : Nitrato (mg $\mathrm{NO}_{3} / \mathrm{L}$ ); PSolu: Fósforo soluble (mg P/L); PTotal: Fósforo total (mg P/L).

\begin{tabular}{|c|c|c|c|c|c|c|c|}
\hline \multicolumn{2}{|c|}{} & FeDisu & Namon & NTK & NO $_{3}$ & PSolu & PTotal \\
\hline \multirow{4}{*}{ AM } & $\mu$ & 6,76 & 0,15 & 1,29 & 0,79 & 0,01 & 0,05 \\
\cline { 2 - 8 } & Min-max & $(0,11-14,09)$ & $(0,06-0,34)$ & $(0,34-2,66)$ & $(0,41-1,26)$ & $(0,00-0,04)$ & $(0,01-0,09)$ \\
\cline { 2 - 8 } & C.V. & 0,81 & 0,69 & 0,59 & 0,38 & 1,54 & 0,69 \\
\hline \multirow{4}{*}{ PG } & $\mu$ & 0,33 & 0,21 & 0,92 & 1,06 & 0,01 & 0,05 \\
\cline { 2 - 8 } & Min-max & $(0,00-1,649)$ & $0,00-0,71)$ & $(0,07-3,17)$ & $(0,22-4,95)$ & $(0,00-0,04)$ & $(0,00-0,21)$ \\
\cline { 2 - 8 } & C.V. & 1,71 & 1,21 & 0,96 & 1,25 & 1,54 & 1,26 \\
\hline \multirow{3}{*}{ PL } & $\mu$ & 0,70 & 0,13 & 1,06 & 0,60 & 0,01 & 0,04 \\
\cline { 2 - 8 } & Min-max & $(0,00-1,96)$ & $(0,0000,65)$ & $(0,14-1,60)$ & $(0,22-0,97)$ & $(0,00-0,04)$ & $(0,00-0,11)$ \\
\cline { 2 - 8 } & C.V. & 1,14 & 1,30 & 0,39 & 0,37 & 1,76 & 0,71 \\
\hline
\end{tabular}




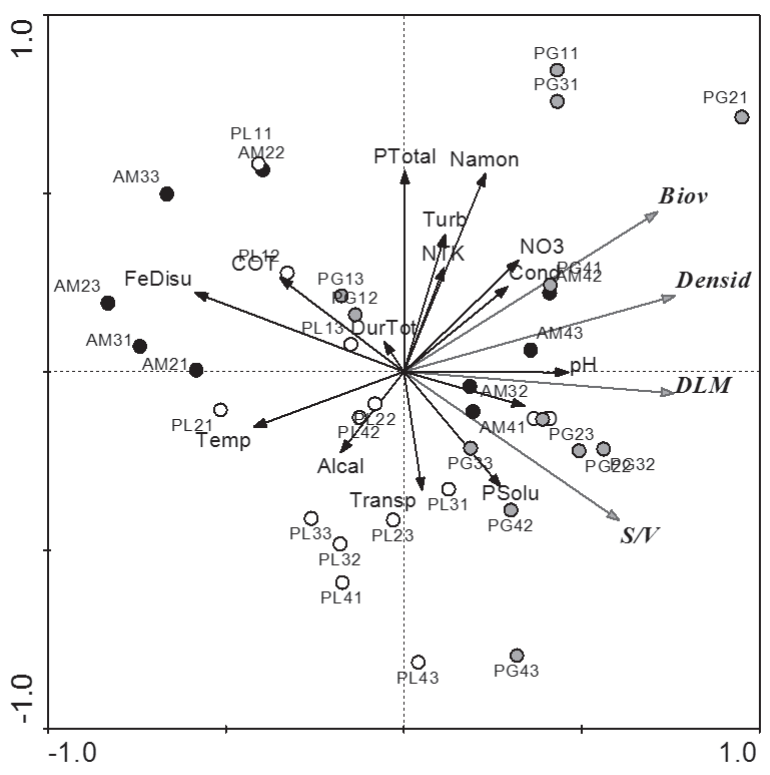

Figura 1. RDA biplot entre épocas. Las estaciones están nombradas según el embalse: AM: Embalse Abreo - Malpaso; PG: Embalse Peñol - Guatapé; PL: Embalse Playas. El primer número hace referencia a la campaña de muestreo, mientras que, el segundo, a la estación.

\section{Rasgos morfológicos del fitoplancton}

Biovolumen geométrico y densidad de organismos: Para evaluar el estado ambiental de un sistema acuático son utilizadas diferentes variables, entre ellas, algunas de carácter biológico, que comprometen el biovolumen geométrico, basado en la morfología de las algas; esta variable representa un factor altamente potente para evaluar la ecología y los patrones de distribución de las comunidades fitoplanctónicas, considerando el volumen de las formas dimensionales, en combinación con el conteo al microscopio. Los resultados correspondientes al biovolumen geométrico de los organismos fitoplanctónicos analizados para cada uno de los embalses, se presentan en la figura 2. Inicialmente, al aplicar la prueba no paramétrica Kruskal - Wallis, se encontró que, a pesar de las observaciones, no existen diferencias estadísticamente significativas entre los embalses estudiados y sus diferentes estaciones ( $p>0,05)$, puesto que la prueba realiza una comparación, a través de la mediana.

Para el embalse Abreo - Malpaso, no se reportaron organismos en ninguna de las estaciones estudiadas en las primeras campañas de muestreo; sin embargo, para los últimos muestreos realizados, se identificaron organismos en las estaciones correspondientes al centro del embalse y salida de aguas, segunda y tercera estación, siendo esta última, la estación donde se registró la mayor cantidad de células por unidad de volumen, con valores cercanos a 40cél/ $\mathrm{mL}$, así como el máximo valor de biovolumen geométrico, para este embalse (valores cercanos a $7 \mathrm{~mm}^{3} / \mathrm{L}$ ) (Figura 2a).

Por otro lado, en el embalse El Peñol - Guatapé, para la primera campaña de muestreo, se registró la mayor densidad de organismos del estudio, encontrándose en la primera estación una densidad total de 120 cél/mL, resaltando, de manera importante, la abundancia de algas en el ingreso de aguas del río Nare a este embalse (Figura 2b), donde se identificó un desarrollo masivo de cianobacterias, predominando formas coloniales con células esféricas embebidas en un mucílago y con elevadas dimensiones, que contribuyeron significativamente al aumento de la densidad de organismos en el embalse y lo que finalmente se tradujo en altos valores de biovolumen, en este caso, con valores máximos cercanos a $20 \mathrm{~mm}^{3} / \mathrm{L}$, claramente asociados al "bloom" de cianobacterias, identificadas como complejo Microcystis.

El complejo Microcystis (Kützing) Kützing presentó la mayor cantidad de biomasa calculada en este embalse, constituyendo alrededor del 78,44\% del biovolumen total, siendo el género de mayor representatividad en todo el estudio. Se resalta, además, la presencia de otros organismos del phylum Cyanobacteria, como los géneros Aphanocapsa, Woronochinia y la especie Radiocystis fernandoi Komárek \& Komárková-Legnerová.

Finalmente, con relación al embalse Playas (Figura 2c), se determinó una mayor densidad de organismos en la primera estación, con valores máximos cercanos a $25 \mathrm{cél} / \mathrm{mL}$, considerablemente menor con respecto a los resultados mencionados anteriormente, que se confrontan, de manera positiva, con los bajos valores de biovolumen geométrico obtenidos para este sistema, con valores máximos cercanos a $0,3 \mathrm{~mm}^{3} / \mathrm{L}$. La mayor parte del fitoplancton encontrado en este sistema acuático estuvo representada por el género Ceratium de la clase Dinophyceae y la cianobacteria del género Chroococcus, esta última, formando colonias relativamente pequeñas, con un número reducido de células y formas esféricas, lo cual, explica los bajos valores de biovolumen registrados en este embalse, en comparación con los otros embalses en estudio. 


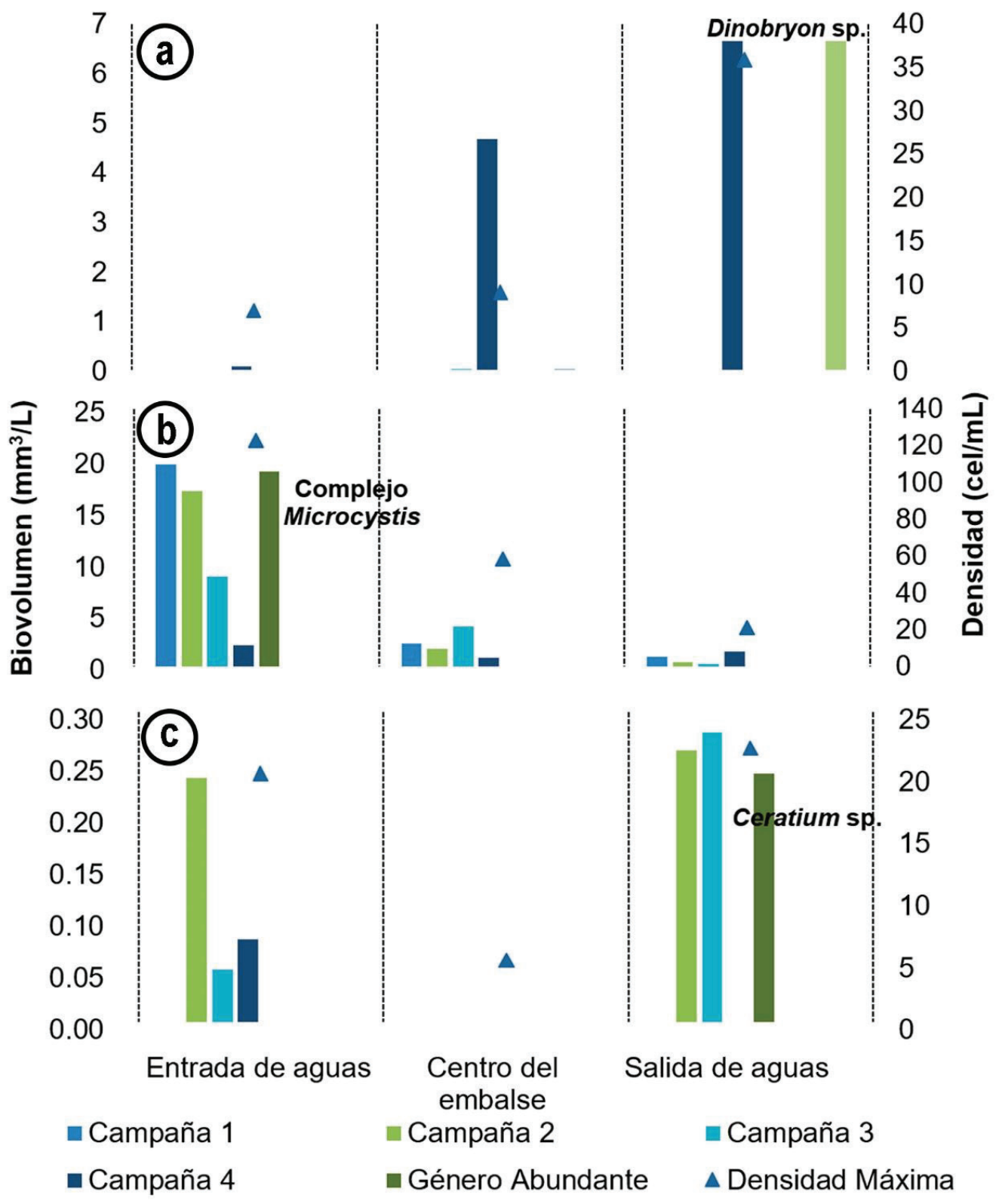

Figura 2. Biovolumen y densidad de organismos en los tres embalses en los Abreo - Malpaso (A), Embalses El Peñol - Guatapé (B) y Playas (C).

Relación superficie volumen $(\mathrm{S} / \mathrm{V})$ y dimensión lineal máxima (DLM): En el embalse Abreo - Malpaso, los valores más altos para la relación $\mathrm{S} / \mathrm{V}$ correspondieron a la primera estación, con un valor promedio de $0,11 \mu \mathrm{m}^{-1}$, como se observa en la tabla 3. Con relación a la DLM, se identificaron organismos con valores hasta de $348 \mu \mathrm{m}$, explicadas, principalmente, por la presencia de organismos de los géneros Oscillatoria y Dinobryon y sus formas geométricas alargadas.

En contraste, en el embalse El Peñol - Guatapé, la mayor relación $\mathrm{S} / \mathrm{V}$ correspondió a la tercera estación (salida de aguas), con un valor medio de $0,19 \mu^{-1}$. Por otro lado, en la estación correspondiente al ingreso de aguas, de especial interés por el "bloom" de cianobacterias evidenciado, se encontró la menor relación $\mathrm{S} / \mathrm{V}$, con un valor medio de $0,16 \mu \mathrm{m}^{-1}$, relacionados, precisamente, con el predominio de cianobacterias, identificadas como complejo Microcystis que, si bien presentaron los valores más altos de biovolumen, también reportaron los valores más bajos de esta relación, debido a su forma geométrica esférica y compacta, que presenta altos valores de volumen y una menor superficie. En cuanto a la DLM, los mayores valores de esta variable correspondieron al fitoplancton identificado en el ingreso de aguas del río Nare, con valores medios de, aproximadamente, $184 \mu \mathrm{m}$, relacionado nuevamente con la presencia del complejo Microcystis. 
Tabla 3. Valores medios ( $\mu$ ), máximos y mínimos (max-min) y coeficiente de variación (C.V.) para las variables morfológicas, relación superficie volumen (S/V) y dimensión lineal máxima (DLM), en los tres embalses (AM: Embalse Abreo - Malpaso; PG: Embalse Peñol - Guatapé; PL: Embalse Playas).

\begin{tabular}{|c|c|c|c|c|c|c|c|}
\hline \multirow{2}{*}{ Estación } & \multirow{2}{*}{ Estadísticos } & \multicolumn{3}{|c|}{$\mathrm{S} / \mathrm{V}\left(\mu \mathrm{m}^{-1}\right)$} & \multicolumn{3}{|c|}{$\operatorname{DLM}(\mu \mathrm{m})$} \\
\hline & & $\mathrm{AM}$ & PG & PL & $\mathrm{AM}$ & PG & PL \\
\hline \multirow{3}{*}{1} & $\mu$ & 0,11 & 0,16 & 0,24 & 74,80 & 184,96 & 43,60 \\
\hline & Min-max & ---- & $0,01-0,58$ & $0,03-0,64$ & ---- & $26,00-435,00$ & $40,70-199,80$ \\
\hline & C.V. & --- & 1,27 & 0,96 & --- & 0,72 & 0,58 \\
\hline \multirow{3}{*}{2} & $\mu$ & 0,07 & 0,18 & ----- & 125,77 & 143,27 & ----- \\
\hline & Min-max & $0,02-4,68$ & $0,02-0,73$ & ---- & $129,50-307,10$ & $19,80-344,10$ & ----- \\
\hline & C.V. & & 1,19 & ----- & 0,38 & 0,65 & ----- \\
\hline \multirow{3}{*}{3} & $\mu$ & 0,02 & 0,19 & 0,36 & 86,95 & 98,73 & 69,18 \\
\hline & Min-max & ---- & $0,05-0,70$ & $0,13-0,58$ & ---- & $35,15-162,80$ & $51,80-114,70$ \\
\hline & C.V. & ---- & 0,89 & 0,60 & ---- & 0,44 & 0,32 \\
\hline
\end{tabular}

Finalmente, para la variable relación S/V en el embalse Playas, los mayores valores se encontraron en el ingreso de aguas, con valores máximos de $0,24 \mu \mathrm{m}^{-1}$, representando los valores más altos reportados en el estudio, relacionados con los géneros Chroococcus y Ceratium. Por último, los mayores valores de DLM en este embalse corresponden a la tercera estación, con valores cercanos a $44 \mu \mathrm{m}$; sin embargo, las dimensiones del fitoplancton de este cuerpo de agua fueron bajas, en comparación con los otros dos sistemas estudiados.

Niveles de riesgo planteados: La literatura indica que el ensamblaje fitoplanctónico responde a los cambios en las condiciones ambientales por medio de la variación interespecífica adaptativa del tamaño, la forma, la organización en estructuras y la capacidad pigmentaria. La diversidad morfométrica, calculada como la relación $\mathrm{S} / \mathrm{V}$ y la DLM, puede estar relacionada con adaptaciones fisiológicas, como la concentración y disposición de pigmentos y la variación en la incorporación de luz y nutrientes (Keddy, 1992; Huszar \& Caraco, 1998).

El cálculo de la relación $\mathrm{S} / \mathrm{V}$ permite elaborar aproximaciones de los procesos metabólicos de los organismos, así como de su interacción con el medio, captación de nutrientes y pérdida de energía; por ejemplo, organismos de menores dimensiones, generalmente, poseen una relación S/V más ventajosa (Margalef, 2002), posibilitando, de esta forma, intercambios con el medio de maneras favorables, traducidas en una actividad metabólica considerable y un crecimiento potencial elevado (Hernández et al. 2012). Del mismo modo, una dimensión lineal máxima podría favorecer el desarrollo del organismo, en términos de captación de luz y de evasión a la herbivoría, constituyendo una ventaja ecológica para su desarrollo, características que pueden significar una potencial proliferación, en condiciones favorables.

Con el fin de establecer una relación entre las tres variables morfológicas: biovolumen geométrico, relación S/V y DLM, se planteó una caracterización del posible riesgo por presencia de cianobacterias para los tres cuerpos de agua, teniendo como valor de referencia los valores máximos y mínimos de estas variables, obtenidas en los tres embalses. Se establecieron cinco diferentes niveles de riesgo, de acuerdo con la interacción de las variables analizadas, como se describe en el cuadro 1.

En el embalse Abreo - Malpaso, debido a la proliferación de plantas acuáticas y la coloración oscura de sus aguas, los organismos encontrados se caracterizaron por poseer formas alargadas y grandes dimensiones, lo que constituye una ventaja ecológica para la captación de luz y posible permanencia en los ecosistemas (Cuadro 1). De igual manera, en la primera estación del embalse El Peñol Guatapé, las altas concentraciones de nitrógeno y fósforo, además de una baja incidencia de corrientes de viento que puedan generar movimiento en esta zona del embalse, favorecen la proliferación de cianobacterias, predominando, por lo tanto, formas coloniales, con células esféricas, embebidas en un mucílago y con elevadas dimensiones. Finalmente, en el embalse Playas, no se determinó una posible floración de cianobacterias, encontrándose, por lo común, organismos de bajas dimensiones, pero que presentaban altos valores de relación $\mathrm{S} / \mathrm{V}$, lo que podría llegar a favorecer el aumento de estos organismos fitoplanctónicos en este sistema, en el caso tal, en que la disponibilidad de nutrientes y de energía lumínica también lo favorezca (Cuadro 1).

Según los niveles de riesgo planteados para los tres embalses de estudio, en los embalses Abreo - Malpaso y Playas, no se encontró una presencia importante de cianobacterias en el cuerpo de agua; sin embargo, los pocos organismos hallados presentaron valores altos de DLM, en el caso del primer embalse o, una alta relación $\mathrm{S} / \mathrm{V}$, para el segundo. Por esto, se sugiere implementar protocolos para la detección de posibles cianotoxinas disueltas en el agua y el monitoreo constante para el control de la proliferación de cianobacterias. 
Cuadro 1. Descripción de los niveles de riesgo planteados para los embalses estudiados.

\begin{tabular}{|c|c|}
\hline $\begin{array}{l}\text { Nivel de } \\
\text { riesgo }\end{array}$ & Descripción del riesgo \\
\hline Bajo & $\begin{array}{l}\text { Fitoplancton con biovolumen reducido, baja relación S/V y DLM, que limitan su interacción con el medio y disminuyen sus ven- } \\
\text { tajas ecológicas para la persistencia en el sistema acuático (Hernández, 2012). }\end{array}$ \\
\hline Medio-Bajo & $\begin{array}{l}\text { Fitoplancton con alto biovolumen, pero con bajos valores de relación S/V y DLM. Fitoplancton de bajo tamaño con una impor- } \\
\text { tante proliferación, que se esperaría no persista en el cuerpo de agua, debido a su baja interacción con el medio y la disminución } \\
\text { de posibles ventajas ecológicas para su persistencia. Se puede también relacionar con un bajo biovolumen y DLM, pero con altos } \\
\text { valores de relación S/V o, por el contrario, bajo biovolumen y relación S/V, pero altos valores de DLM que, si bien no indican un } \\
\text { riesgo inminente, puede favorecer una adecuada interacción con su medio o la captación lumínica necesaria, que en eventos futuros } \\
\text { puedan beneficiar su proliferación. Tanto el embalse Abreo - Malpaso como Playas, se encuentran en esta categoría, a pesar de } \\
\text { presentar resultados diferentes en relación a los organismos identificados. }\end{array}$ \\
\hline Medio & $\begin{array}{l}\text { Altos valores de biovolumen con bajos valores de DLM relacionadas con fitoplancton de menor tamaño; sin embargo, presenta } \\
\text { una importante relación S/V, que podría llevarlo al aumento de su densidad al relacionarse positivamente con su medio y poder } \\
\text { captar, de éste, la mayor cantidad de elementos necesarios para proliferar. También, se puede relacionar con bajos valores de bio- } \\
\text { volumen, pero altos valores para relación S/V y DLM, variables que pueden significar una importante ventaja ecológica para } \\
\text { la proliferación de organismos en el embalse. }\end{array}$ \\
\hline Medio-alto & $\begin{array}{l}\text { Fitoplancton con alto valor de biovolumen. Aunque presenta una baja relación S/V, poseen una alta DLM, expresada en organis- } \\
\text { mos fitoplanctónicos de gran tamaño, con importante ventaja ecológica para la captación lumínica y la evasión de diversos procesos } \\
\text { de herbivoría (Hernández, 2012). De acuerdo con las características identificadas en el embalse El Peñol - Guatapé, se puede } \\
\text { encontrar dentro de este nivel. }\end{array}$ \\
\hline Alto & $\begin{array}{l}\text { Nivel de mayor alerta para el sistema acuático. Biovolumen geométrico algal muy alto, con alta relación S/V y DLM. Proliferación } \\
\text { evidente de cianobacterias, que poseen una relación importante con su medio y que le permite el intercambio efectivo de elementos } \\
\text { necesarios para su proliferación, además de poseer grandes dimensiones, que le permiten captar las cantidades de luz necesarias } \\
\text { para su desarrollo masivo y una importante resistencia a procesos de herbivoría (Hernández, 2012). }\end{array}$ \\
\hline
\end{tabular}

Con relación al embalse El Peñol - Guatapé, aunque presentara, en general, menores concentraciones de nutrientes en sus dos últimas estaciones, sí indicó altos valores en la primera estación, con valores máximos de 3,18mg N/L para NTK; 4,96mg $\mathrm{NO}_{3} / \mathrm{L}$ de $\mathrm{NO}_{3}$ y $0,21 \mathrm{mg} \mathrm{P} / \mathrm{L}$, valores altos en comparación con los otros embalses (Tabla 2). Esta primera estación, correspondiente al ingreso del río Nare, constituye el principal riesgo determinado en el estudio, encontrándose un elevado biovolumen de cianobacterias, conformado, principalmente, por colonias identificadas, como complejo Microcystis, con altas dimensiones y un número elevado de células, además de altos valores de NTK, nitrógeno amoniacal, lo cual, indica la necesidad inmediata de protocolos y de medidas de manejo, tanto para el control de la proliferación de estos organismos como para el estudio de posibles cianotoxinas disueltas en el agua.

Se concluye que las características funcionales, incluyendo aspectos morfológicos, pueden aportar conocimiento con relación al rendimiento ecológico de un sistema. La escasa información sobre las características morfológicas y fisiológicas de las especies fitoplanctónicas en Colombia, limita la posibilidad de lograr una clasificación funcional a priori. Es, por esta razón, que los estudios realizados con la implementación de rasgos morfológicos, los cuales, son relativamente fáciles de medir y cuya relación con la fisiología está bien definida, sugieren la utilización de las relaciones entre la morfología y las propiedades funcionales, para determinar aspectos ecológicos de los ecosistemas; de esta forma, la continuidad de asociaciones entre propiedades cuantitativas de los organismos y el diagnóstico de los ecosistemas es pertinente y relevante en el manejo los servicios ecosistémicos del recurso hídrico.

Conflictos de intereses: El manuscrito fue preparado y revisado con la participación de todos los autores, quienes declaramos que no existe ningún conflicto de intereses que ponga en riesgo la validez de los resultados presentados. Financiación: Este estudio fue financiado por la Vicerrectoría de Investigaciones de la Universidad de Antioquia y Empresas Públicas de Medellín (EPM), en la que participaron los grupos Geolimna y GDCON de la misma universidad.

\section{REFERENCIAS}

1. AGUIRRE RAMÍREZ, N.J.; PALACIO BAENA, J.; RAMÍREZ RESTREPO, J. 2007. Características limnológicas del embalse el Peñol-Guatapé, Colombia. Rev. ing. univ. Medellin. (Colombia). 6(10):53-66.

2. BONILLA, S.; HAAKONSSON, S.; SOMMA, A.; GRAVIER, A.; BRITOS, A.; VIDAL, L.; DE LEÓN, L.; BRENA, B.M.; PÍREZ, M.; PICCINI, C.; MARTÍNEZ DE LA ESCALERA, G.; CHALAR, G.; GONZÁLEZ-PIANA, M.; MARTIGANI, F.; AUBRIOT, L. 2015. Cianobacterias 
y cianotoxinas en ecosistemas límnicos de Uruguay. Innotec (Uruguay). 10:9-22. http://dx.doi.org/10.6084/m9.figshare

3. BULA, G. 1985. Florecimientos nocivos de algas verde-azules en dos lagunas del departamento del Magdalena. Rev. Ing. Pes. 6:1-2.

4. CORNARE. 2012. Zonificación de riesgo por movimientos en masa inundación y avenidas torrenciales. Atención de áreas afectadas por eventos desastrosos. Municipio de Rionegro.

5. CORNARE. 2012a. Zonificación de riesgo por movimientos en masa inundación y avenidas torrenciales. Atención de áreas afectadas por eventos desastrosos. Municipio de San Rafael.

6. ESCOBAR, A.; MANJARRES, G. 1985. Estudio de un florecimiento de algas toxicas en la Ciénaga San Rafael de Buena Vista Magdalena Colombia. Rev. Ing. Pesquera. 5:17-37.

7. HERNÁNDEZ, E.; AGUIRRE, N.; PALACIO, J.; RAMÍREZ, J.J.; DUQUE, S.; GUISANDE, C.; MOGOLLÓN, M.; ARANGUREN, N. 2012. Rasgos morfológicos del fitoplancton en seis sistemas leníticos de las regiones Amazónica, Andina y Caribe de Colombia. Act. Biol. (Colombia). 34(96):67-83.

8. HERNÁNDEZ, E.; AGUIRRE, N.J.; PALACIO, J.A. 2011. Relación entre la determinación del pigmento Clorofila a y el Biovolumen geométrico algal en un lago de planicie de inundación (Ciénaga de Ayapel, Córdoba-Colombia). Redin. 60:159-169.

9. HILLEBRAND, H.; DÜRSELEN, C.D.; KIRSCHTEL, D.; POLLINGHER, U.; ZOHARY, T. 1999. Biovolume calculation for pelagic and benthic microalgae. J. Phycol. (Estados Unidos). 35(2):403-424. https://doi.org/10.1046/ j.1529-8817.1999.3520403.x

10. HOFFMANN, L.; KOMÁREK, J.; KAŠTOVSKÝ, J. 2005. System of cyanoprokaryotes (cyanobacteria)-state in 2004. Algol. Stud. (Alemania). 117(1):95-115. https://doi. org/10.1127/1864-1318/2005/0117-0095

11. HUSZAR, V.L.; CARACO, N.F. 1998. The relationship between phytoplankton composition and physical-chemical variables: a comparison of taxonomic and morphological-functional descriptors in six temperate lakes. Freshwater Biology 40(4):679-696. https://doi.org/10.1046/j.1365-2427.1998. d01-656.x

12. KEDDY, P.A. 1992. A pragmatic approach to functional ecology. Functional Ecology. 6(6):621-626.
13. KOMÁREK, J.; ANAGNOSTIDIS, K. 1989. Modern approach to the classification system of Cyanophytes 4-Nostocales. Algol. Stud. (Alemania). 82(3):247-345.

14. KOMÁREK, J.; ANAGNOSTIDIS, K. 1999. Subwasserflora von mitteleuropa: Cyanoprokaryota. Ed. Springer Spektrum (Alemania). 1131p.

15. MANCERA, P.; ERNESTO, J.; VIDAL, V.; ALFONSO, L. 1994. Florecimiento de microalgas relacionado con mortandad masiva de peces en el complejo lagunar Ciénaga Grande de Santa Marta, Caribe colombiano. Boletín de Invest. Marinas y Costeras - INVEMAR. 23(1):103-117. https://doi.org/10.1038/44819

16. MARGALEF, R. 2002. Diversidad y biodiversidad. En: Pineda, J.D.; Miguel, J.M. de; Casado, M.A.; Montalvo, J. (eds.). La diversidad biológica de España. CYTED. Prentice Hall (Edit.) Madrid. p.3-6.

17. OBERHOLSTER, P.J.; BOTHA, A.M.; CLOETE, T. 2006. Toxic cyanobacterial blooms in a shallow, artificially mixed urban lake in Colorado, USA. Lakes Reserv. Res. Manag. (Australia). 11(2):111-123. https://doi.org/10.1111/j.14401770.2006.00297.x

18. OTTEN, T.G.; PAERL, H.W. 2015. Health effects of toxic cyanobacteria in US drinking and recreational waters: our current understanding and proposed direction. Curr. Environ. Health Rep. (Suiza). 2(1):75-84. http://doi. org/10.1007/s40572-014-0041-9

19. PEROVICH, G.Q.; DORTCH, Q.; GOODRICH, J.; BERGER, P.; BROOKS, J.; EVENS, T.J.; GOBLER, C.J.; GRAHAM, J.; HYDE, J.; KARNER, D.; O'SHEA, D.; PAUL, V.; PAERL, H.; PIEHLER, M.; ROSEN, B.; SANTELMANN, M.; TESTER P.; WESTRICK, J. 2008. Causes, Prevention, and Mitigation. In: Hudnell, H.K. (ed). Cyanobacterial Harmful Algal Blooms: State of the Science and Research Needs. SSBM (New York). p.185-215.

20. ROLLAND, D.C.; BOURGET, S.; WARREN, A.; LAURION, I.; VINCENT, W.F. 2013. Extreme variability of cynobacterial blooms in an urban drinking water supply. J. Plankton Res. 35(4):744-758. https://doi.org/10.1093/plankt/fbt042

21. ROS J. 1979. Prácticas de Ecología. Omega. 181p.

22. UHELINGER, V. 1964. E'tude statisque des methodes de de'nobrement planctonique. Arch. Sci. 17:121-123.

23. VÁSQUEZ, C.; ARIZA, A.; PINILLA, G. 2006. Descripción del estado trófico de diez humedales del altiplano cundiboyacense. Universitas Scientiarum. 11(2):61-75. 